\title{
Microring resonator-based diamond optothermal switch: a building block for a quantum computing network
}

Zhihong Huang, Andrei Faraon, Charles Santori, Victor Acosta, Raymond G. Beausoleil

Zhihong Huang, Andrei Faraon, Charles Santori, Victor Acosta, Raymond G. Beausoleil, "Microring resonator-based diamond optothermal switch: a building block for a quantum computing network," Proc. SPIE 8635, Advances in Photonics of Quantum Computing, Memory, and Communication VI, 86350E (29 March 2013); doi: 10.1117/12.2002631

SPIE. Event: SPIE OPTO, 2013, San Francisco, California, United States 


\title{
Microring resonator-based diamond opto-thermal switch - a building block for a quantum computing
}

\author{
Zhihong Huang ${ }^{a}$ and Andrei Faraon ${ }^{a, b}$ and Charles Santori ${ }^{a}$ and Victor Acosta $^{a}$ and Raymond \\ G. Beausoleil ${ }^{a}$ \\ ${ }^{a}$ Intelligent Infrastructure Lab, Hewlett-Packard Labs,Palo Alto, USA; \\ ${ }^{b}$ Applied Physics and Materials Science, California Institute of Technology, Pasadena CA, USA
}

\begin{abstract}
We demonstrate an optical-thermal switch on diamond. The design is based on micro-ring resonator coupled with two waveguides. A drop efficiency of $31 \%$ and through port efficiency of $73 \%$ were achieved.
\end{abstract}

Keywords: Diamond, optical switch, quantum information processing, nitrogen-vacency centers

\section{INTRODUCTION}

The negatively-charged nitrogen-vacancy centers in diamond has motivated many groups building scalable quantum information processors based on diamond photonics. This is owning to the long-lived electronic spin coherence and the capability for spin manipulation and readout of NV centers. ${ }^{1-4}$ The primitive operation is to create entanglement between two NV centers, based on schemes such as 'atom-photon entanglement' proposed by Cabrillo et al. ${ }^{5}$ To scale this type of scheme beyond two qubits, one important component is an optical switch that allows light emitted from a particular device to be routed to multiple locations. With such a switch, one has choices of routing photons to specified paths and has the benefit of improving the entanglement speed by entangling multiple qubits at the same time. Yield of the existing diamond cavities coupled with NV centers are inevitably low, due to the nature of randomness for NV placement and orientation, variation of spectral stability, and variation of cavity resonance frequency and quality factor. An optical switch provides the capability to tolerate a large fraction of defective devices by routing only to the working devices. Many type of switching devices were built on conventional semiconductor materials with mechanisms from mechanical, thermal switching to carrier injection, photonics crystal, and polymer refractive index tuning.$^{6-8}$ In this paper, we build an optical-thermal switch on diamond with micro-ring waveguides, mainly for the simplicity of the diamond fabrication. The the switching function was realized by locally tuning the temperature of the diamond waveguides. Switching efficiency of $31 \%$ at 'drop' port and $73 \%$ at 'through' port were obtained.

\section{DESIGN, EXPERIMENTS AND DISCUSSION}

\subsection{Design and fabrication}

The design of the thermal switch is shown in Fig. 1. The device consists of a diamond micro-ring resonator $(20 \mu \mathrm{m}$ diameter) coupled to two waveguides. When the resonator is in tune with the input optical frequency, light in the straight waveguide couples to the resonator and travels to the 'drop' port. At detuning, light transmits to the 'through' port without coupling to the micro-ring. The switching function is thus realized by thermally shifting the refractive index of diamond to tune the resonant frequencies of the cavity. Due to the present difficulties of fabricating structures in diamond, we use the simplest possible approach for thermal tuning, which is to use an external laser to heat the device. More selective heating is made possible by placing a metallic region within the ring to absorb the incident light. A more integrated design would use an electrically driven heater instead of laser heating, and in fact electrical heaters are commonly integrated into similar devices made in silicon.

Since the thermal refractive index shift in diamond is small $\left((1 / n) d n / d T \approx 3.2 \times 10^{-6}\right.$ at $\left.300 \mathrm{~K}^{9}\right)$, large temperature shifts are needed for tuning. The temperature has to raise above $300 \mathrm{~K}$ to observe effective tuning

Further author information: (Send correspondence to Z. Huang)

Z. Huang: E-mail: zhihong.huang@hp.com, Telephone: 16508573209

Advances in Photonics of Quantum Computing, Memory, and Communication VI, Zameer U. Hasan,

Philip R. Hemmer, Hwang Lee, Charles M. Santori, Eds., Proc. of SPIE Vol. 8635, 86350E

(c) 2013 SPIE · CCC code: 0277-786X/13/\$18 · doi: 10.1117/12.2002631 


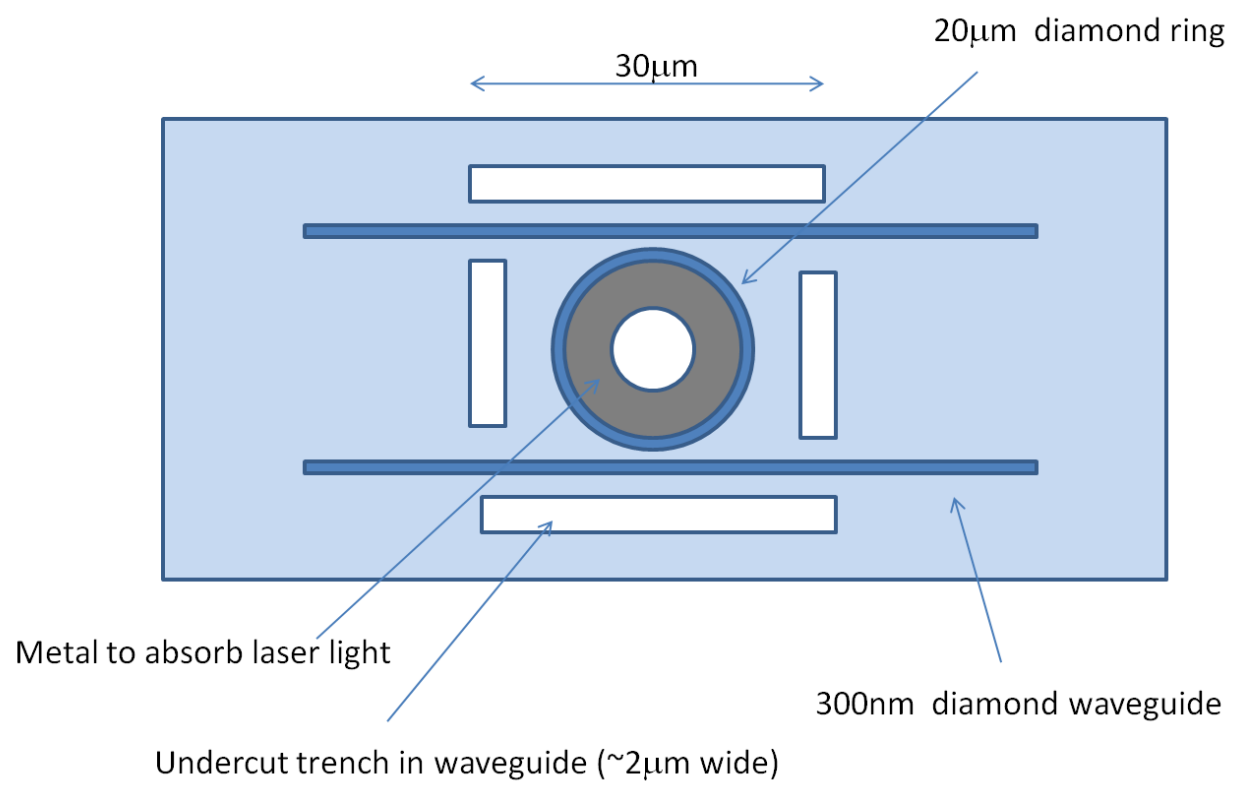

Figure 1. Schematic diagram of a thermally tunable switch in diamond, based on a micro-ring resonator coupled to two waveguides.

of the switch. On the other hand, the NV centers comprising the qubits must be kept below $10 \mathrm{~K}$ to avoid phonon-induced broadening of the optical transition. ${ }^{10}$ Thus, the thermal switch has to be isolated from the cavities containing NV centers. To implement thermal isolation, we first place the diamond membrane on a thick thermal $\mathrm{SiO}_{2}$ on silicon, further undercut $\mathrm{Si}$ beneath the structure to let the resonator resting on thermal $\mathrm{SiO}_{2}$ only.

A typical fabrication process is shown in Fig. 2. We started with a $5 \mu \mathrm{m}$-thick polished diamond membrane obtained commercially from element-6. We placed the membrane on a $2 \mu \mathrm{m}$-thick thermal oxide on silicon and defined the microring and waveguide structures with e-beam lithography with a negative resist (FOX-12 Dow Corning), following by another e-beam lithography to define the $500 \mathrm{~nm}$ chrome metal ring for heat absorption, as shown in Fig.1. To isolate the switch from the underneath silicon, we then etched trenches with inductivelycoupled-plasma reactive-ion-etcher (ICP-RIE) into oxide and silicon over $10 \mu \mathrm{m}$-deep, and selectively undercut the underneath silicon using dry or wet etching. The diamond structure, along with the supporting $\mathrm{SiO}_{2}$ will be free-standing on air and supported by a few pillars surrounding the structure region.

We performed a finite-element simulation with COMSOL heat transfer module, and the results are shown in Fig. 3. Due to the small refractive index shift of diamond, in order to obtain a sufficient wavelength shifts, it is desired to raise the temperature of diamond above $300 \mathrm{~K}$. The simulation indicates that $7 \mathrm{~mW}$ of heating power is sufficient to heat the structure to $390 \mathrm{~K}$, even by placing the sample at $5 \mathrm{~K}$. In the simulation, because of the isolation of the structure, the heating is rather localized within the device (Fig. 3), and the temperature increase is negligible beyond $\sim 50 \mu \mathrm{m}$ of distance. This is partly a result of the efficient heat spreading in the silicon substrate. Similar simulation shows that a device with no the thermal isolation trench requires $10 \times$ power to obtain $390 \mathrm{~K}$ around the diamond ring.

Fig. 4 shows images of a fabricated device after defining diamond waveguides, prior to heater and thermal isolation. The device is resting on $\mathrm{SiO}_{2}$ layer. The sidewall of the diamond waveguide are smooth with a $\sim 100 \mathrm{~nm}$ gap clearly resolved between each straight waveguide and the micro-ring resonator. The linear waveguide was designed narrower than the ring waveguide to improve the mode matching. ${ }^{11}$ Grating couplers are places at the end of the straight waveguide for normal collection of photons in the free-space microscopy setup. 
(a)

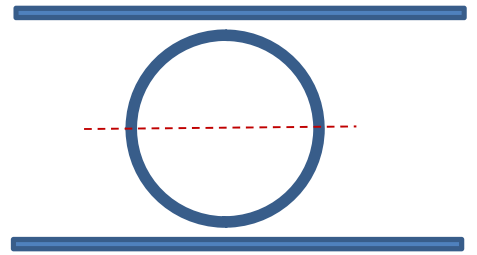

(b)

Diamond $(5 \mu \mathrm{m})$
$\mathrm{SiO}_{2}$
$\mathrm{Si}$

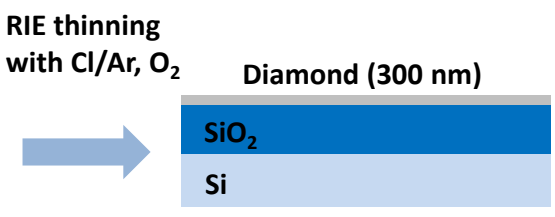

Spin resist

(Fox-12)

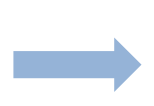

Etch
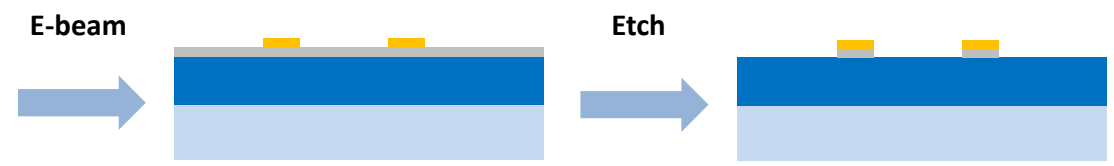

Remove

resist
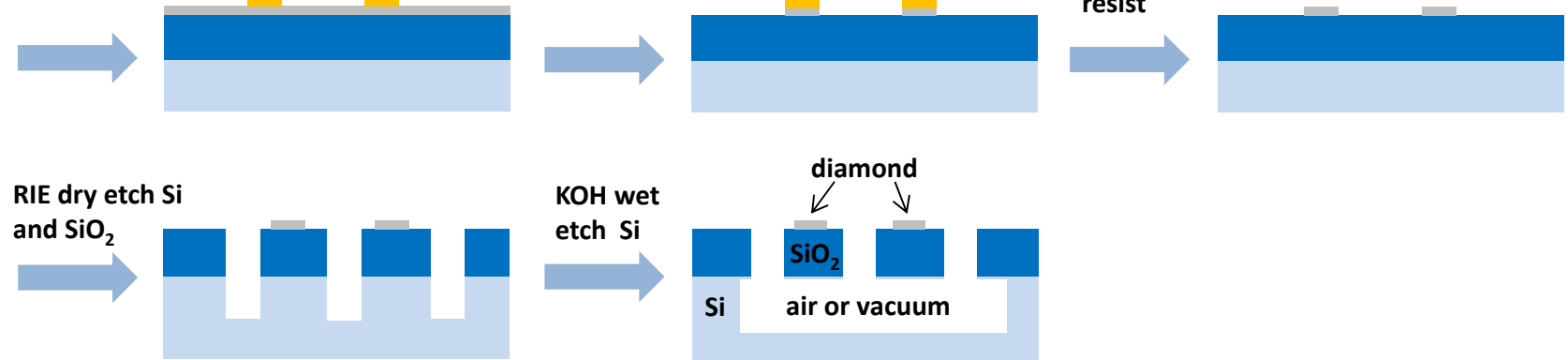

$\mathrm{KOH}$ wet

etch Si

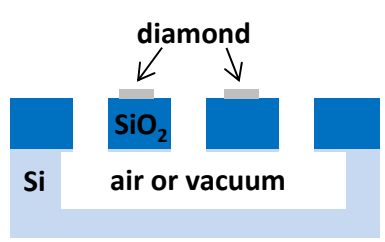

Figure 2. (a) The red-dashed line indicates the cross-sectional direction used for the diagrams below. (b) The initial fabrication process used to make the thermally tunable switch.

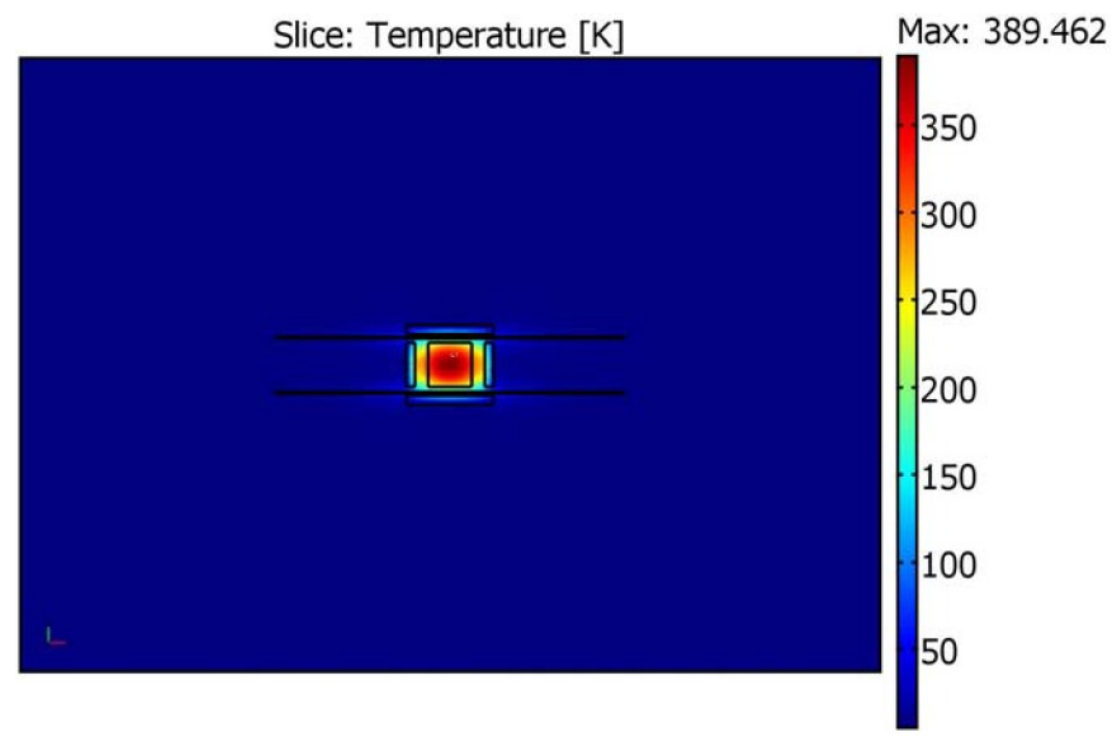

Min: 5.00

Figure 3. Finite element simulation (Comsol) of the temperature profile of the micro-ring thermal switch. The graph scaling is set to the full range, showing that a device temperature of $390 \mathrm{~K}$ can be reached for $7 \mathrm{~mW}$ of heating power. 


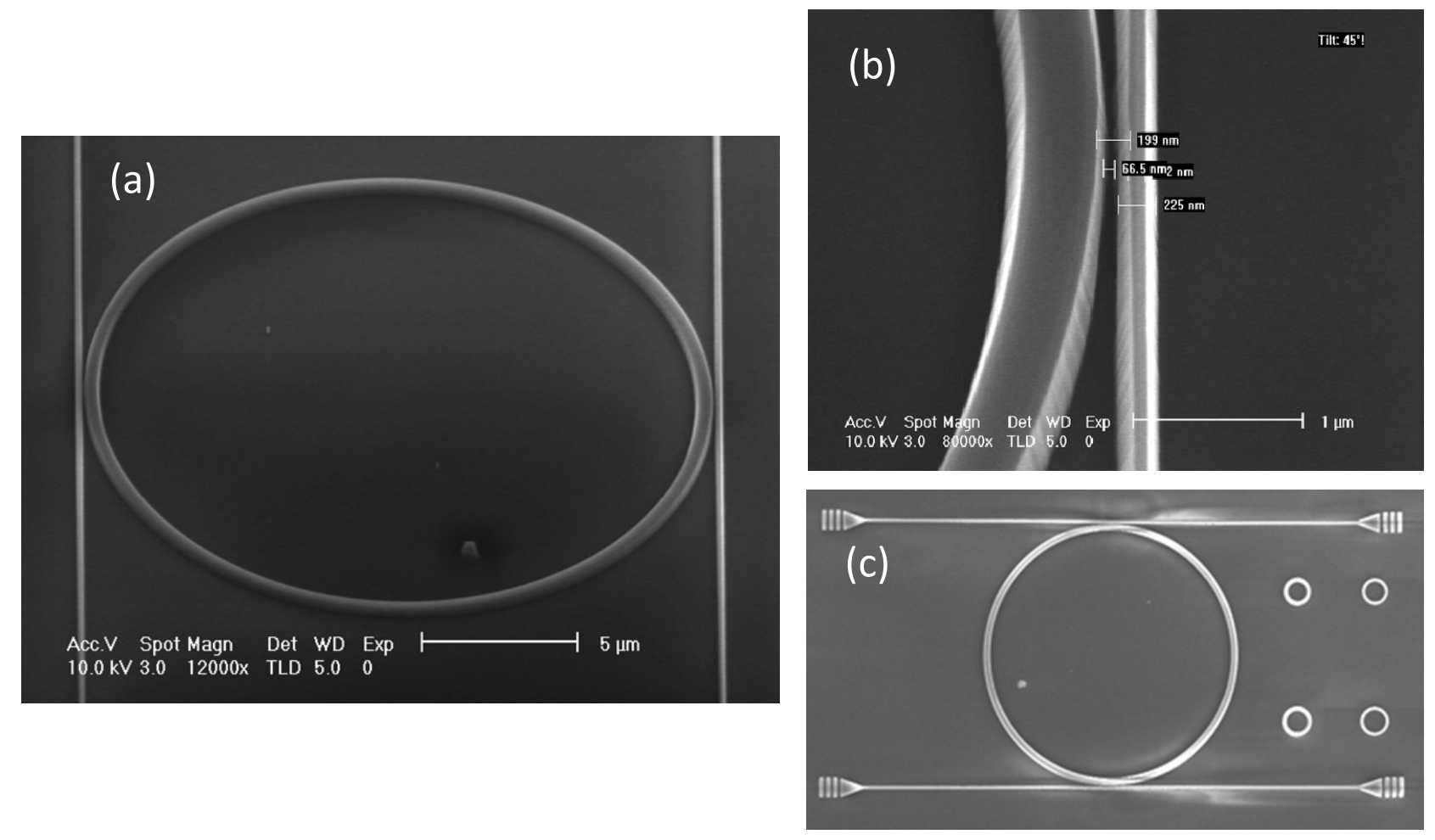

Figure 4. Scanning-electron-microscope images of a fabricated structure before undercut: (a) A diamond ring resonator coupled to two waveguides, (b) Close-up of one of the coupling regions, and (c) the entire circuit, including grating couplers.

\subsection{Switch efficiencies}

After etching the diamond waveguides, we tested the thermal switching mechanism by heating the entire chip on a sample mount. Transmission measurements were performed by focusing a supercontinuum laser onto the 'input' grating, and collecting the light exiting vertically either from the 'drop' (Fig. 5(a)) or 'through' (Fig. 5(b)) gratings, which were aligned with a movable pinhole, as shown in Fig. 5(c). The collection efficiency theoretically is independent of sample orientation due to the symmetric design of the structure, consistent with well processed devices. The cavity resonances appear as a peak at the 'drop' port and a dip at the 'through' port, as only light at resonant frequencies can be coupled to the resonator and travel to the 'drop' port, while light at other frequencies only transmit to the 'through' port. The quality factor $\mathrm{Q}$ of the cavities is $\sim 3000$ and the free spectral range of the device is $2.4 \mathrm{~nm}$, determined primarily by the ring circumference. The transfer efficiency into the drop waveguide can approach $100 \%$ only when the scattering loss in the resonator is much smaller than the coupling rates to the waveguides. Shallower dip contrasts at the 'through' port are due to scattering loss from sidewall roughness, curvature radiation loss, and coupling loss between the micro-ring and the two straight waveguides. By increases from $320-400 \mathrm{~K}$, the resonant peak of the device has a red shift as temperature, with a total shifts approximately 1.5 linewidths, as shown in 5(b). We estimate the drop efficiency to be $32 \%$. Our model predicts that for $0.33 \mathrm{~nm}$ of tuning off of resonance, we should expect $T_{\text {through }}=0.73$. Switching efficiencies can be further improved by reducing scattering and coupling losses.

\subsection{Discussion}

We next attempt to create trench and undercut region needed for thermal isolation, as outlined in Fig. 2. The sample was first deposited $1 \mu \mathrm{nm}$-thick PECVD oxide to protect the surface, followed by a deep trench etch over $10 \mu \mathrm{m}$ in RIE. Silicon was selectively wet etched in $30 \%$ w.t. $\mathrm{KOH}$ solution at $80^{\circ} \mathrm{C}$ along (100) plane, at a rate of $1 \mu \mathrm{m}$ per minute. The complete undercut was completed in 7 minutes, as shown in Fig. 6(a). However, diamond waveguides appeared peeling off after 5 minutes exposure to the solution which is shown in Fig. 6(b). 

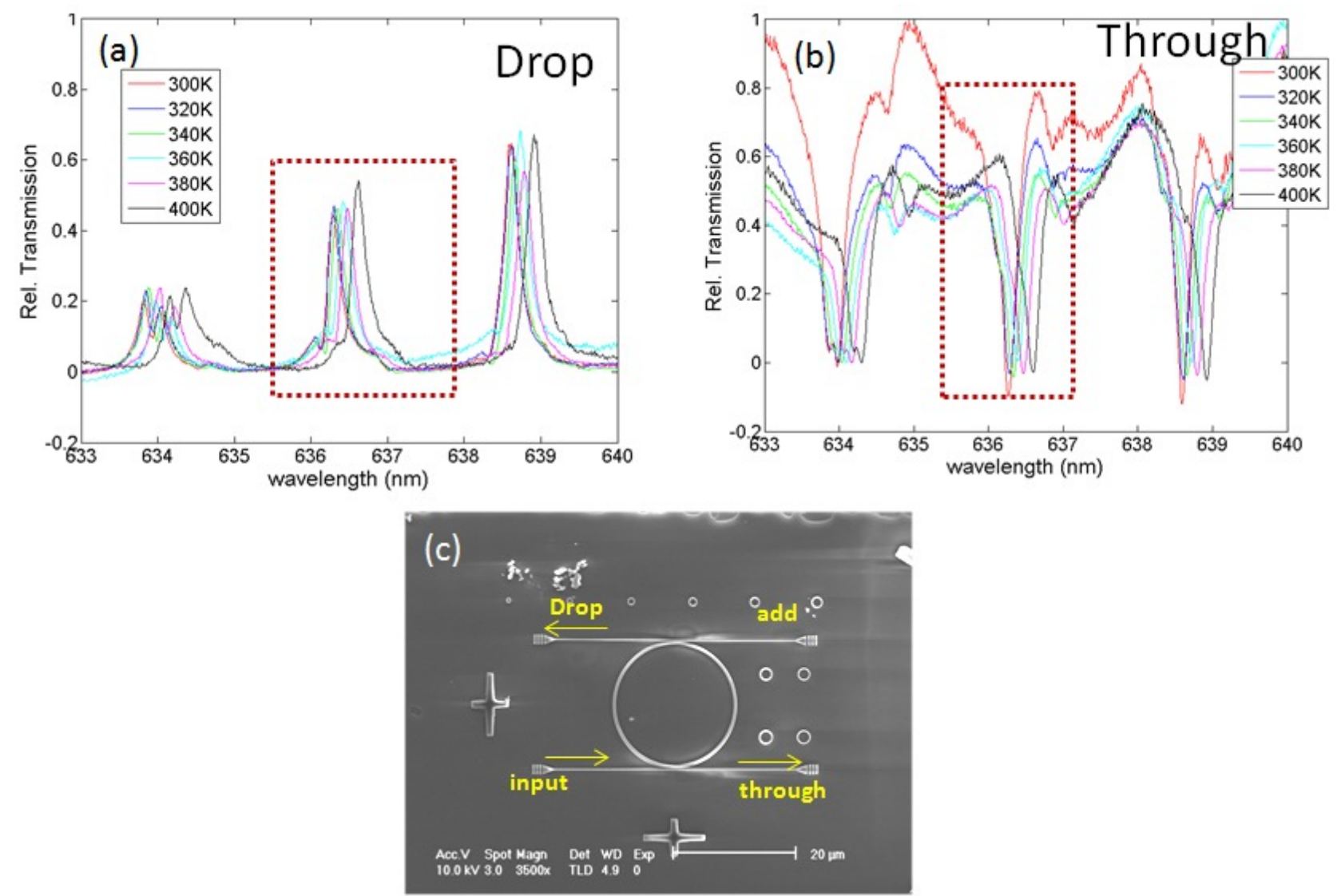

Figure 5. Thermal tuning results obtained by heating the entire sample: (a) Transmission measurements collecting from the 'drop' port, showing how the transmission peaks associated with cavity modes shift with temperature. (b) Transmission measurements collecting from the 'through' port, showing how the transmission dips associated with cavity modes shift with temperature. (c) Diagram to illustrate how the measurements were performed using free-space excitation and collection through the grating couplers. 

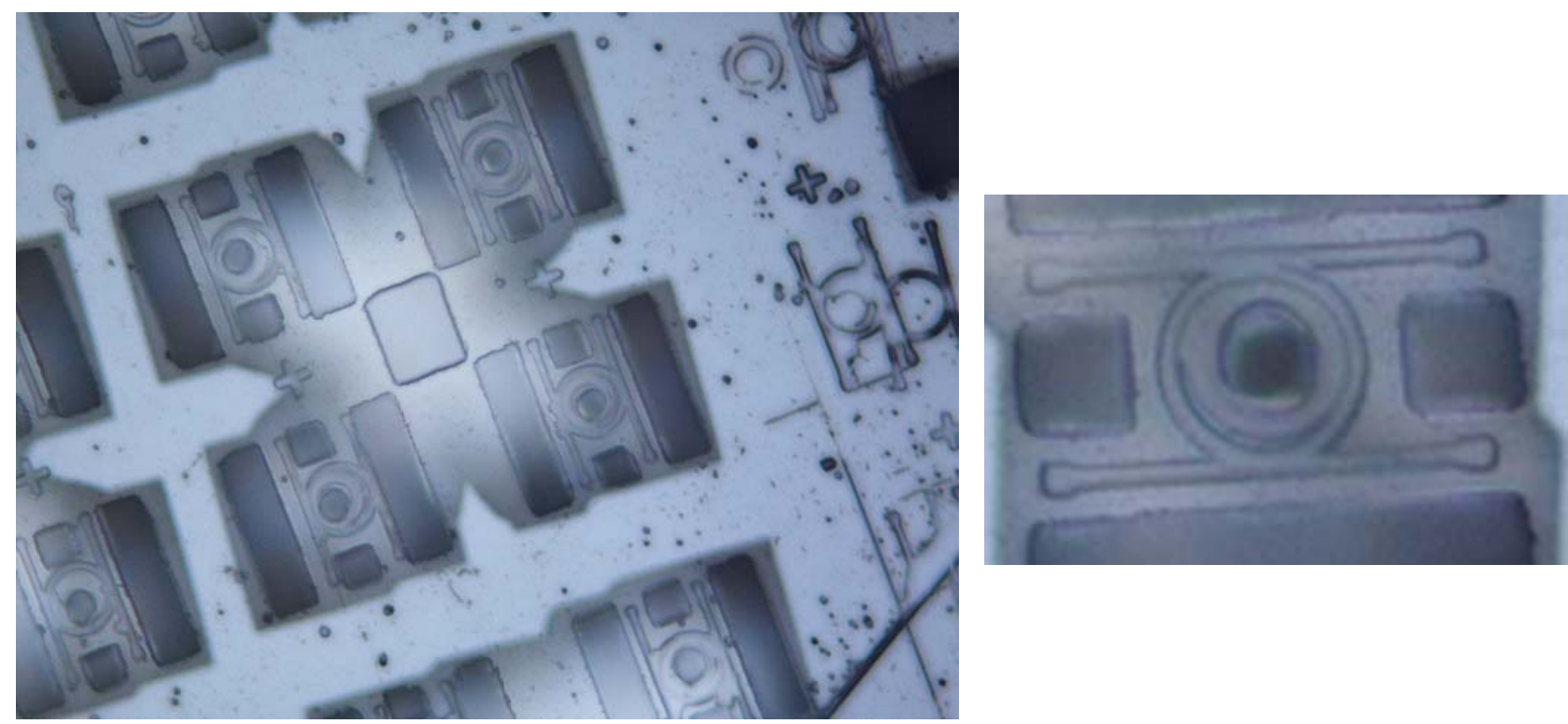

Figure 6. Optical microscope images (Nomarski mode) of thermal switch devices following the final KOH wet etch used to create the undercut regions, following our original recipe.

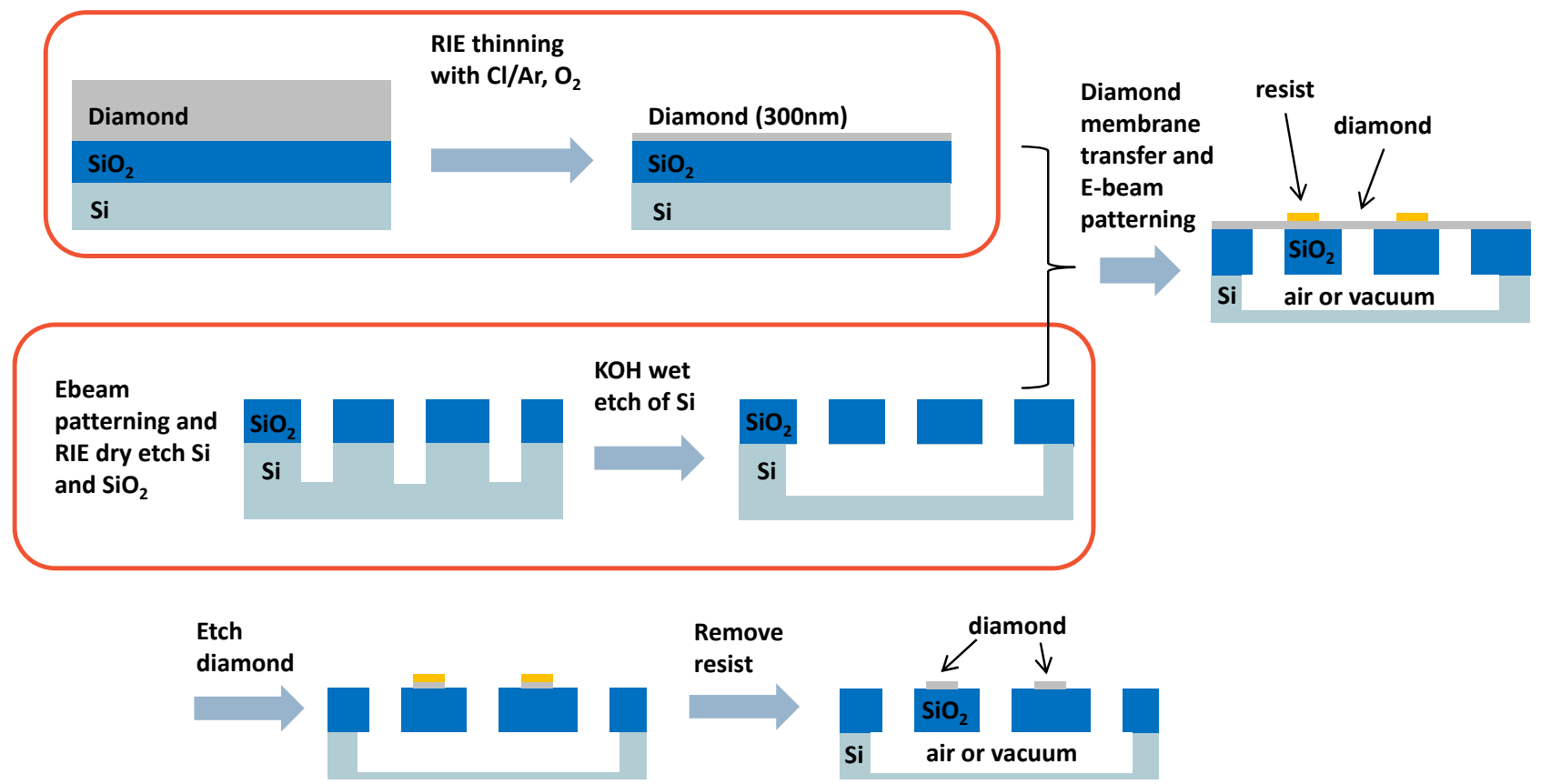

Figure 7. New fabrication process for obtaining thermally isolated switches in diamond. The trenches and undercut regions are created before a diamond membrane is transferred to the $\mathrm{Si} / \mathrm{SiO}_{2}$ substrate. The diamond photonic elements are created last. 
We speculate that this is due to the chemical reaction between $\mathrm{KOH}$ and $\mathrm{SiO}_{2}$. Although $\mathrm{KOH}$ is expected to have an etch selectivity as high as 600:1 between $\mathrm{Si}$ and $\mathrm{SiO}_{2}, \mathrm{KOH}$ may attack the vulnerable side-walls around the waveguide sufficiently to cause the diamond layer to detached from the $\mathrm{SiO}_{2}$ layer underneath.

As an alternative, we modify the process: create the undercut on the substrate first, thin the diamond a separate carrier, and place them on the undercut substrate to define the waveguide by e-beam lithography through alignment. The new processing is shown in Fig. 7 and the procedures are under testing.

\section{ACKNOWLEDGMENTS}

This manuscript is based on work supported by the Defense Advanced Research Projects Agency (award no. HR0011-09-1-0006) and The Regents of the University of California.

\section{REFERENCES}

[1] Gruber, A., Dräbenstedt, A., Tietz, C., Fleury, L., Wrachtrup, J., and von Borczyskowski, C., "Scanning Confocal Optical Microscopy and Magnetic Resonance on Single Defect Centers," Science 276, 2012-2014 (June 1997).

[2] Jelezko, F., Gaebel, T., Popa, I., Domhan, M., Gruber, a., and Wrachtrup, J., "Observation of Coherent Oscillation of a Single Nuclear Spin and Realization of a Two-Qubit Conditional Quantum Gate," Physical Review Letters 93, 1-4 (Sept. 2004).

[3] Jelezko, F., Gaebel, T., Popa, I., Gruber, a., and Wrachtrup, J., "Observation of Coherent Oscillations in a Single Electron Spin," Physical Review Letters 92, 1-4 (Feb. 2004).

[4] Childress, L., Taylor, J. M., Sorensen, A. S., and Lukin, M. D., "Fault-tolerant quantum communication based on solid-state photon emitters," Phys. Rev. Lett. 96, 70504 (2006).

[5] Cabrillo, C., Cirac, J. I., García-Fernández, P., and Zoller, P., "Creation of entangled states of distant atoms by interference," Phys. Rev. A 59, 1025-1033 (Feb 1999).

[6] Notomi, M., Shinya, A., Mitsugi, S., Kira, G., Kuramochi, E., and Tanabe, T., "Optical bistable switching action of si high-q photonic-crystal nanocavities," Optics Express 13(7), 2678-2687 (2005).

[7] Toshiyoshi, H. and Fujita, H., "Electrostatic micro torsion mirrors for an optical switch matrix," Microelectromechanical Systems, Journal of 5(4), 231-237 (1996).

[8] Sasaki, K. and Nagamura, T., "Ultrafast wide range all-optical switch using complex refractive-index changes in a composite film of silver and polymer containing photochromic dye," Journal of applied physics 83(6), 2894-2900 (1998).

[9] Ruf, T., Cardona, M., Pickles, C., and Sussmann, R., "Temperature dependence of the refractive index of diamond up to $925 \mathrm{k}, "$ Physical Review B 62(24), 16578 (2000).

[10] Fu, K.-M. C., Santori, C., Barclay, P. E., Rogers, L. J., Manson, N. B., and Beausoleil, R. G., "Observation of the Dynamic Jahn-Teller Effect in the Excited States of Nitrogen-Vacancy Centers in Diamond," Phys. Rev. Lett. 103(25), 256404 (2009).

[11] Xu, Q., Fattal, D., and Beausoleil, R. G., "Silicon microring resonators with 1.5- $\mu$ m radius," Opt. Express 16(6), 4309-4315 (2008). 